\title{
Editorial
}

\section{Nutraceuticals: Recent Advances of Bioactive Food Components}

\author{
Pyo-Jam Park, ${ }^{1}$ Thomas Ty Wang, ${ }^{2}$ Eun-Kyung Kim, ${ }^{3}$ and Qian Zhong-Jii \\ ${ }^{1}$ Department of Biotechnology, Konkuk University, Chungju 380-701, Republic of Korea \\ ${ }^{2}$ Diet, Genomics and Immunology Lab, Beltsville Human Nutrition Research Center, United States Department of Agriculture, \\ Beltsville, MD 20705, USA \\ ${ }^{3}$ Division of Food Bio Science, College of Biomedical and Health Sciences, Konkuk University, Chungju 380-701, Republic of Korea \\ ${ }^{4}$ Food Science and Technology, Guangdong Ocean University, Zhanjiang 524088, China \\ Correspondence should be addressed to Pyo-Jam Park; parkpj@kku.ac.kr
}

Received 24 January 2016; Accepted 26 January 2016

Copyright (C) 2016 Pyo-Jam Park et al. This is an open access article distributed under the Creative Commons Attribution License, which permits unrestricted use, distribution, and reproduction in any medium, provided the original work is properly cited.

Nutraceuticals are numerous and have been studied intensely for basic science and applied research. The term is generally used to refer to those chemicals that may have biological significance, for example, antioxidants, disease resistance, and regulating the immune systems, but are not established as essential nutrients. It utilizes traditional and rapidly advancing analytical methods and instrumentation such as differential solvent extraction, gas or liquid chromatography coupled with tandem mass spectrometry, nuclear magnetic resonance and electron-spin resonance, and gene and protein arrays for fractionation, purification, chemical and genetic fingerprinting, derivatization, modification, synthesis, and clinical trials of photochemicals starting from cell-line to animal and patient studies. The goal of this special issue is to provide a platform for scientists all over the world to promote, share, and discuss various new issues and developments in the area of nutraceuticals research.

This issue compiles biological activities of bioactive food components.

Kombucha is described in a review and research paper. Kombucha tea is produced by fermenting sugared black tea with a mixed culture of yeast and bacteria. Kombucha tea has gained immense popularity in recent times due to many associated health benefits. The therapeutic effects of this beverage are thought to be derived from the chemical composition of this beverage, mainly the polyphenols and secondary metabolites which are produced during fermentation. The stability of antioxidant activity and polyphenol contents were also investigated by M. I. Watawana et al.
Calcium compounds of Opuntia ficus-indica are reported by I. Rojas-Molina et al. from Mexico which have a deficiency in calcium intake. They characterize the distribution of calcium compounds in soluble and insoluble dietary fiber extracted from Opuntia ficus-indica.

Enriching pasta with grape marc is studied by V. Marinelli et al. from Italy. Grape marc is an interesting source of natural compounds as polyphenols and fibers. This is the study to enrich fresh and dry pasta with grape marc extracts obtained by means of UAE using only water as solvent extraction.

Antioxidant peptide is obtained from Spirulina maxima by W. S. Kim et al. This paper deals with the antioxidant peptide derived from Spirulina maxima possessing an effective constituent in antitumor progression products based on the mechanism of tumor progression through ROS and the HIFl $\alpha$ signaling pathway.

Tunisian Cupressus sempervirens chemical composition and some biological activities are analyzed by A. B. Nouri et al. Some of the studies already focused on chemistry and biological activities of Cupressus sempervirens; however they are originated from different areas in the world. This study is to ascertain the chemical composition of Tunisian Cupressus sempervirens to evaluate its antioxidant and antibacterial activities.

4-(4-Methylbenzamino)benzoate is observed to suppress adipocyte differentiation by J. T. Hwang et al. 4(4-Methylbenzamino)benzoate has estimated adipogenesissuppressing activity compared to resveratrol or genistein. 
The biologically active substances of regrowth velvet antler are described in this issue. In this study, regrowth velvet antler is subjected to extraction by DW to allow determination of its constituent biologically active substances, including uronic acid, sulfated GAGs, sialic acid, uracil, hypoxanthine, and uridine. In addition, the antioxidant activities of RVA are determined by assessing DPPH, $\mathrm{H}_{2} \mathrm{O}_{2}$, hydroxyl, and ABTS radical scavenging activity as well as FRAP and ORAC.

We thoroughly reviewed these papers and we believe that they contribute to the applications of bioactive food components. Moreover, we believe that the papers in this special issue will be milestones for the advancement for the next generation of nutraceuticals researchers.

Pyo-Jam Park Thomas Ty Wang Eun-Kyung Kim Qian Zhong-Ji 

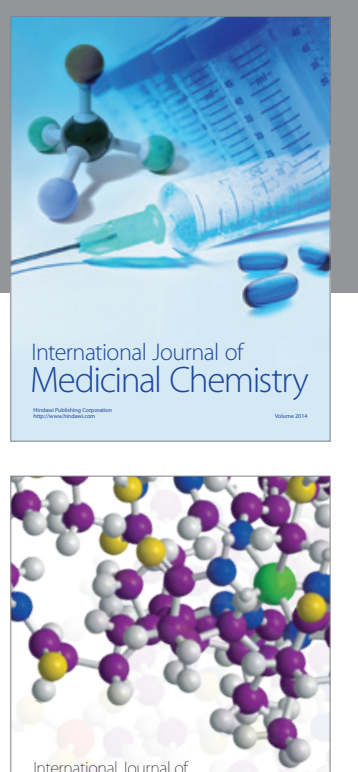

Carbohydrate Chemistry

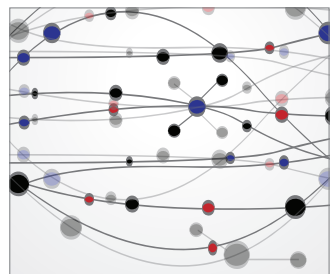

The Scientific World Journal
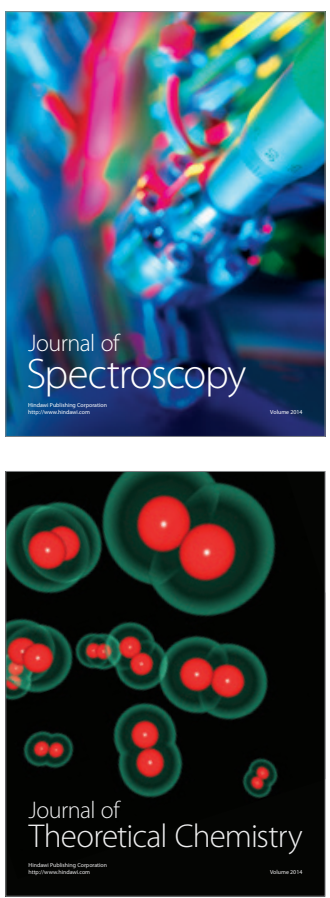
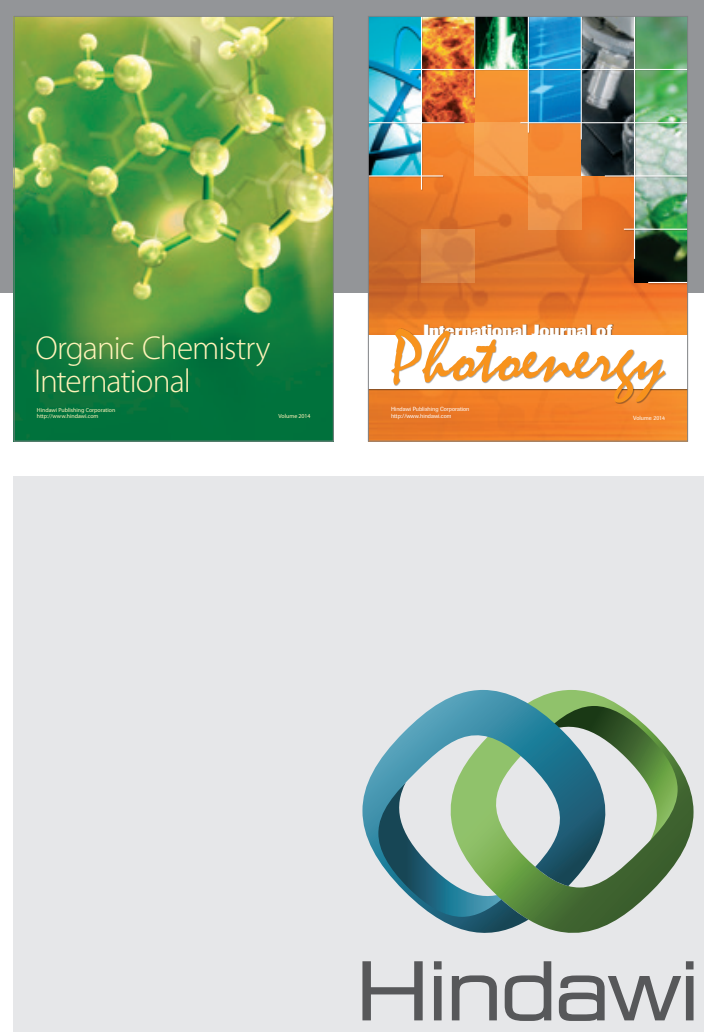

Submit your manuscripts at

http://www.hindawi.com

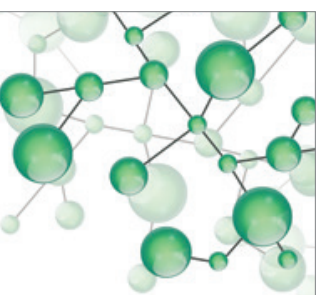

International Journal of

Inorganic Chemistry

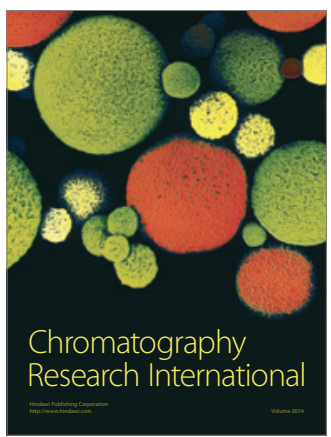

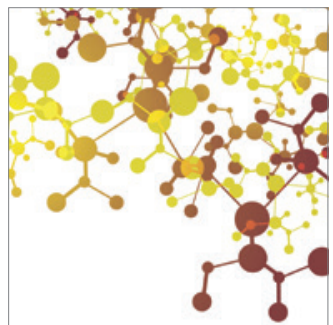

Applied Chemistry
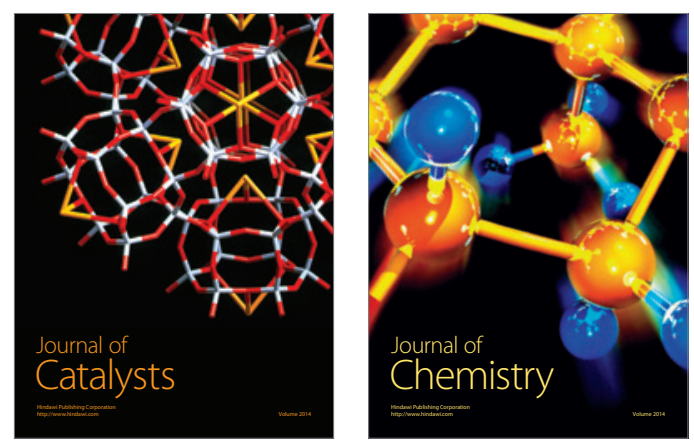
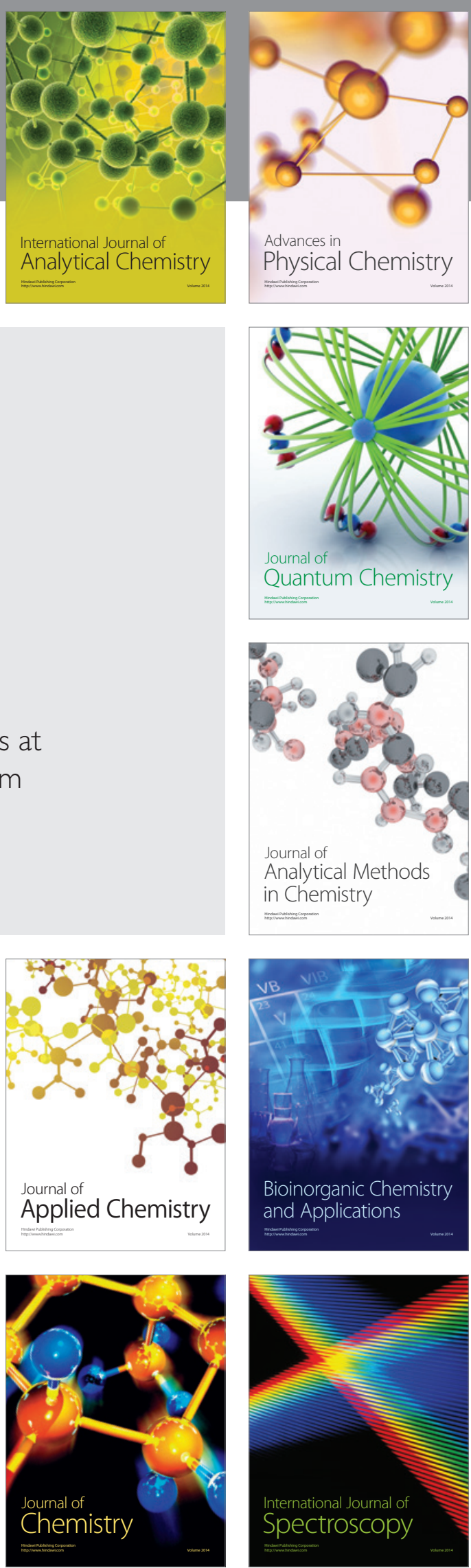